\title{
Articuler des analyses qualitatives et quantitatives pour mieux comprendre l'activité in situ : deux études en éducation physique et en sport
}

\author{
Gilles Kermarrec, Ghada Regaieg et Rebecca Clayton-Bernard
}

\begin{abstract}
Gilles Kermarrec.
Ghada Regaieg.

Rebecca Clayton-Bernard.

Résumé. Pour appréhender des phénomènes complexes in situ, des chercheurs adoptent des méthodes qualitatives. Ce chapitre présente une méthode mixte articulant des analyses qualitatives et quantitatives : (1) une catégorisation qualitative empirique ou théorique ; (2) le codage quantitatif de l'activité ou le recueil de données quantitatives sur l'activité ; (3) une analyse statistique croisant des variables catégorielles et des variables numériques. Deux études sur l'apprentissage et la prise de décision sont présentées pour illustrer comment une méthode mixte a permis : (1) de valider la catégorisation d'unités de sens ; (2) d'évaluer la puissance discriminatoire des catégorisations qualitatives. Les méthodes mixtes pourraient ainsi contribuer à en améliorant la compréhension de l'activité in situ des pratiquants ou des intervenants.
\end{abstract}

Mots-clés. Méthode mixte, Activité, Cognition située, Stratégies d’apprentissage, Décision

\section{Introduction}

Même si les méthodes mixtes tentent de concilier des méthodes qualitatives et des méthodes quantitatives, celles-ci sont souvent juxtaposées. Les méthodes qualitatives explorent un phénomène et offrent des hypothèses à corroborer ensuite à l'aide de méthodes quantitatives (Greene, 2007). Ce chapitre montre l'intérêt d'une véritable intégration d'analyses qualitatives et quantitatives pour conforter ou interroger des systèmes de catégorisation issus d'analyses qualitatives.

Dans les sciences de l'intervention en Éducation Physique et en Sport, pour appréhender la complexité des phénomènes étudiés, de nombreux chercheurs ont adopté des méthodes qualitatives. Considérant la cognition comme située (Hauw, 2018), ils visent la description de l'activité d'un acteur en situation naturelle, dans un cadre scolaire (Vors, Gal-Petitfaux \& Potdevin, 2015) ou compétitif (Kerivel et al., 2020).

D’un point de vue méthodologique, les chercheurs analysent l'activité en situation, en assumant une certaine « subjectivité » : (1) en accordant un primat au point de vue des acteurs sur leurs propres expériences ; (2) en procédant à des catégorisations empiriques ou théoriques qui ne peuvent s'affranchir du point de vue des chercheurs. En effet, les efforts méthodologiques pour asseoir la validité des catégories issues de leurs analyses reposent principalement sur une procédure de triangulation (Kerivel et al., 2020). Celle-ci consiste à effectuer une analyse indépendante de tout ou partie du corpus de données par des chercheurs différents. Néanmoins, ces chercheurs mènent leur travail avec des cadres d'analyse homogènes, car ils sont souvent impliqués dans une même équipe de recherche, ou dans un même courant de pensée (le cadre théorique et méthodologique de l'analyse sémiologique du cours d'action).

Aussi, nous avons eu la curiosité de confronter des variables catégorielles issues d'analyses qualitatives, à des analyses statistiques reposant sur un codage quantitatif d'un aspect du phénomène étudié. Nous illustrerons nos propos avec deux études, l'une portant sur les stratégies d'apprentissage mobilisées par des élèves lors de situations d'apprentissage en Éducation Physique et Sportive (Kermarrec, Todorovitch \& Fleming, 2004), l'autre s'intéressant à la prise de décision d'un gardien de but de hand-ball, en situation de compétition (Le Men, Kermarrec, Bossard, Travassos \& Duarte, 2019). L'objectif de ce chapitre est de présenter de façon détaillée la méthodologie, en l'illustrant pas à pas, 
Articuler des analyses qualitatives et quantitatives pour mieux comprendre l'activité i...

afin de montrer comment en intégrer des analyses qualitatives et quantitatives. Aussi, nous avons introduits au fur et à mesure de la méthode, des éléments de résultats de ces deux études, afin qu'ils illustrent la démarche.

\section{Méthodologie}

L'étude de la cognition en situation constituait l'objectif de ces deux études. Plus précisément, la première étude a investigué les stratégies d'apprentissage utilisées par 23 élèves issus de quatre classes de collège en France, dans quatre situations d'apprentissage en EPS (recevoir et renvoyer un ballon en volley-ball; varier les trajectoires du volant en dégagé en badminton; varier les trajectoires de balle en service en tennis de table; se balancer à la barre fixe en gymnastique), La seconde étude a examiné les prises de décision d'un gardien de but professionnel, lors de 26 situations de tir d'un match de première division du championnat de France. La méthode utilisée dans ces deux études repose sur cinq étapes.

Des données comportementales ont été recueillies en filmant les situations. Des données verbales ont été recueillies lors d'un entretien avec un rappel stimulé de l'expérience vécue, en présentant le film de la situation au participant. Les relances portaient sur l'activité du participant au présent : ici que fais-tu ? Et maintenant que ressens- tu ? Là que penses-tu ? Que regardes-tu ? Cette technique de recueil de donnée se rapproche de celle de l'entretien d'autoconfrontation, issu de la méthode d'analyse du cours d'action (Theureau, 2010). Avec ce recueil de données, nous disposions d'un corpus d'unités significatives relatives à l'activité in situ : 56 comportements et verbatim relatifs à des stratégies d'apprentissage des 23 élèves en EPS, 83 portions de discours relatives aux prises de décision d'un gardien de but au cours d'un match.

Pour réaliser une catégorisation qualitative le chercheur identifie dans les données d'observation et / ou verbales, des segments ou " unités de sens » en relation avec son objet d'étude. Ces unités de sens sont ensuite progressivement classées, regroupées en fonction de similitudes et de différences sémantiques identifiées par le chercheur. Pour cela, soit il s'appuie sur un modèle théorique (catégorisation théorique) ou travaille sans modèle a priori dans la perspective d'une théorisation ancrée (catégorisation empirique). Lors de l'étude des stratégies d'apprentissage en EPS, nous avons adopté pour une catégorisation empirique, car nous ne disposions pas de cadre théorique éprouvé dans le contexte de l'EPS. L’analyse de contenu a permis d'identifier 56 unités significatives, regroupés en six catégories de stratégies d’apprentissage (répéter ; focaliser son attention ; réfléchir et comprendre ; observer-imiter ; imager-simuler ; écouter les consignes).

Pour l'étude de la prise de décision, nous avons utilisé d'une part une catégorisation théorique fondée sur le modèle de la prise de décision par reconnaissance (Klein, 2015). La pertinence de modèle pour caractériser les processus décisionnels en sports collectifs avait été confortée dans plusieurs études précédentes (en football par Kermarrec \& Bossard, 2014 ; en volley-ball par Macquet, 2009). Trois processus sont distingués (la «reconnaissance simple» et le choix immédiat de la première option, la «simulation mentale» de la première option pour l'évaluer, et le « diagnostic » c'est-à-dire la comparaison d'options plausibles). D’autre part, une catégorisation empirique a été utilisée pour caractériser les contenus des prises de décision, car ces contenus étaient considérés comme particulièrement spécifiques à ce poste de gardien de but. Quatre types de contenus décisionnels ont été identifiés (identifier la position adaptée dans le but par rapport à l'évolution du jeu ; identifier un tireur potentiel ; identifier la zone accessible pour le tireur ; identifier la fin de la trajectoire du tir).

Cette étape peut consister à coder de façon quantitative un aspect du phénomène initialement analysé de façon qualitative (Cleary, Zimmerman \& Keating 2006) ou à recueillir des données quantitatives complémentaires, et relatives au même phénomène (Gal-Petitfaux, Adé, Poizat \& Seifert, 2013). Dans la situation d'apprentissage, il s'agissait de comptabiliser pour chaque élève le nombre d'occurrences des unités significatives considérées comme des manifestations des stratégies d'apprentissage. Plus un élève exprimait verbalement lors d'un entretien qu'il « observe 
attentivement un pair », qu'il « fait attention à la démonstration de l'enseignant », plus le nombre d'occurrences de la stratégie « observer - imiter » augmentait, et ceci pour les 23 participants. Ainsi, nous disposions de 205 occurrences des 56 unités significatives des stratégies d’apprentissage utilisées par des élèves en EPS.

Le chercheur peut aussi « compléter » son recueil par des mesures « objectives » portant sur un ou plusieurs aspects du phénomène étudié. En hand-ball, lors de chaque action, nous avons recueilli la distance gardien de but -tireur potentiel, car cette distance constituait un indicateur plausible de la pression temporelle susceptible d'affecter la prise de décision (Klein, 2015). Cette mesure a été effectuée à l'aide d'un système de vidéo-tracking, au moment de chaque prise de décision du gardien de but. Nous disposions alors 83 mesures de la distance gardien de but-tireur potentiels.

Des analyses statistiques peuvent viser soit la confirmation de la catégorisation qualitative, soit l'évaluation de sa puissance discriminatoire. A ces fins des analyses factorielles discriminantes ont été utilisées dans la première étude. Des comparaisons de moyennes inter-groupes et des tests statistiques associés ont été utilisés dans la seconde étude. Dans la section suivante nous allons présenter les résultats de cette articulation entre analyse qualitative et quantitative.

\section{Résultats et discussion}

Dans l'étude des stratégies d'apprentissage en EPS, nous disposions pour 23 participants de 205 occurrences de 56 unités significatives, classées a priori dans 6 catégories empiriques. Par hypothèse, la répartition des occurrences de chaque moyen d'apprendre n'était pas aléatoire, mais dépendait de tendances, d'habitudes de travail issues des expériences passées et singulières des différents élèves en EPS. Aussi, des moyens d'apprendre (verbatim et comportements) regroupés ensemble dans une même catégorie de stratégie d'apprentissage devraient se retrouver plus souvent chez un même sujet. Une analyse factorielle discriminante a confirmé le classement statistique de 54 des 56 unités significatives en 6 catégories de stratégies d’apprentissage (figure 1). 
Articuler des analyses qualitatives et quantitatives pour mieux comprendre l'activité i...

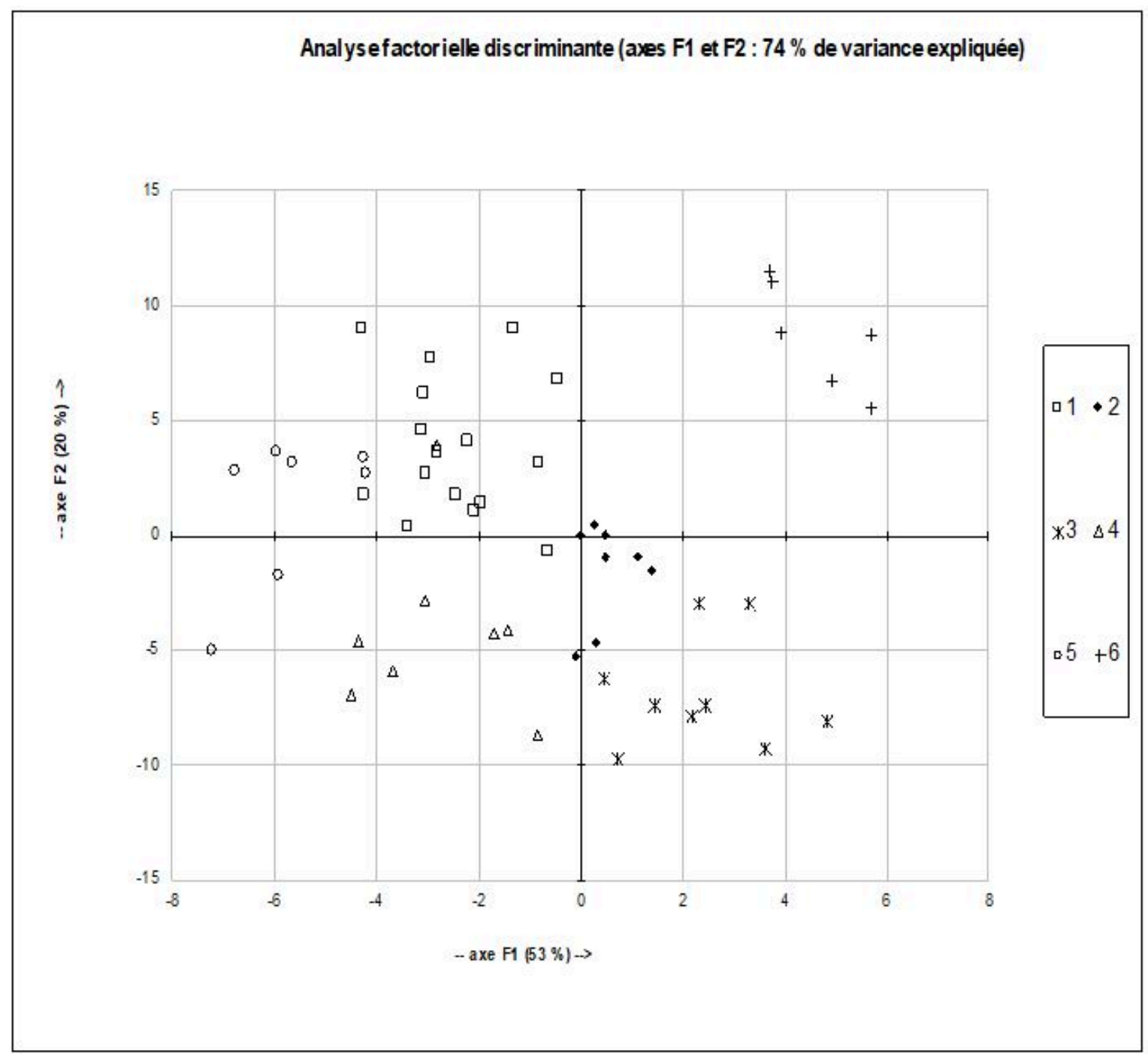

Figure 1 : Répartition des unités significatives par l'analyse factorielle discriminante

(1) Écouter les consignes ; (2) Analyser et comprendre ; (3) Observer et insister ; (4) Simuler ou imaginer l'action ;

(5) Focaliser son attentionnelle ; (6) Répéter

Dans l'étude sur les stratégies d'apprentissage, la distribution des unités significative se traduit par la position de variables regroupées en classes dans un plan factoriel. L'analyse discriminante recherche les combinaisons linéaires qui séparent le mieux les catégories les unes des autres. Le lambda de Wilks est calculé pour évaluer la puissance discriminatoire du modèle (valeur de 1 , aucune discrimination, à 0 , parfaite discrimination) : ici, Lambda de Wilks $=0,002 ; \mathrm{F}(115 ; 142)=3,012, \mathrm{p}<.001$. Complémentairement, la comparaison par paires des distances inter-classes montre par exemple que des élèves qui utilisent la stratégie de répétition peuvent utiliser conjointement l'écoute des consignes, mais qu'ils n'utilisent alors pas la stratégie de focalisation de l'attention, ce qui conforte l'hypothèse de profil d’apprentissage (Kermarrec \& Michot, 2007 ; Roure, Kermarrec \& Pasco, 2019)

Dans l'étude de la prise de décision chez un gardien de but de haut-niveau, chaque unité de sens recueillie «en première personne » pouvait être associée une donnée quantitative recueillie " en troisième personne ». On pouvait alors calculer une distance moyenne gardien-tireur associée à chaque catégorie qualitative. Des comparaisons de 
Actes de la 11ème Biennale de l'ARIS : Former des citoyens physiquement éduqués. Axe 2 - Vers une éducation physique de qualité, 10.25518/sepaps20.426

moyennes inter-catégories ont été réalisées à l'aide du test $\mathrm{H}$ de Kruskal-Wallis. Les distances moyennes associées aux trois catégories de processus (1. Reconnaissance simple, 7,17 $\pm 3,13 \mathrm{~m} ; 2$. Simulation mentale, 7,81 $\pm 6,06 \mathrm{~m}$; 3. Diagnostic, 9,15 $\pm 6,94 \mathrm{~m}$ ) n'étaient pas significativement différentes. Par contre, les distances moyennes associées aux quatre catégories de contenus de prise de décision (1. identification de la position adaptée dans le but, 11,61 \pm $11,26 \mathrm{~m} ; 2$. identification du tireur, $10,42 \pm 5,13 \mathrm{~m} ; 3$. identification de la zone accessible pour le tireur, 6,03 $\pm 2,38$ $\mathrm{m} ; 4$. identification de la fin de la trajectoire du tir, 5,52 $\pm 2,79 \mathrm{~m}$ ) étaient significativement différentes : $\chi^{2}(3)=$ $26,53, \mathrm{p}<.001$.

Dans la première catégorisation, la relation entre les processus de reconnaissances, du plus rapide au moins rapide, et les distances moyennes est cohérente d'un point de vue théorique. Néanmoins, les analyses statistiques montrent que cette catégorisation des expériences n'est pas discriminante au regard de la variable distance gardien-tireur. Par contre, la seconde catégorisation montrent qu'il y aurait une relation entre les contenus des décisions d'un gardien de hand-ball, tels qu'ils sont décrits par notre système de catégorisation, et la distance gardien-tireur. Pour comprendre comment un gardien de but de hand-ball de haut-niveau fait face aux contraintes spatio-temporelles lors du tir, une catégorisation de ses expériences en terme de contenus de la prise de décision serait donc plus pertinente qu'une catégorisation des expériences en terme de processus de reconnaissance. Plus précisément, pour gérer l'urgence ou la pression temporelle, un gardien de but expert en hand-ball procède par micro-décisions successives, au fur et à mesure que le tireur se rapproche de lui (Le Men, Kermarrec, Bossard, Travassos \& Duarte, 2019).

\section{Conclusion}

Les deux études présentées illustrent la complémentarité des méthodes qualitatives et quantitatives, car une approche de l'activité humaine à partir du point des acteurs (first person approach) serait pertinente pour décrire leurs expériences in situ, alors qu'une approche de l'activité à partir de données extrinsèques (third-person approach) permet de mettre en évidence aspects du phénomène qui ne peuvent pas être spontanément décrits par ces mêmes acteurs (Hauw et al., 2017). Aussi, le chercheur peut soit recourir à un codage quantitatif des données qualitatives recueillies (nombre d'occurrences), soit à un recueil de données quantitatives complémentaires. Ce chapitre montre une facette de l'étendue du spectre des méthodes mixtes. Trois types de méthodes mixtes sont généralement distinguées en fonction de la façon dont les données qualitatives et quantitatives sont intégrées (Guetterman, Fetters \& Creswell, 2015 ; Greene, 2007 ; Tashakkori \& Teddlie, 2010) : (1) l'intégration par connexion de phases (méthodes et résultats sont présentés séparément, avant interprétation) ; (2) l'intégration par comparaison de résultats (collectes séparées et analyses séparées ou interdépendantes ; (3) l'intégration par assimilation des données (transformation de données qualitatives en données quantitatives ou inversement). Dans ce chapitre, l'étude des stratégies d'apprentissage a reposé sur une quantification des données qualitatives (type 3), alors que l'étude de la prise de décision a mobilisé une comparaison de résultats en interrogeant leur interdépendance (type 2).

Finalement ce chapitre montre comment une analyse quantitative permet de conforter ou de discuter les catégories issues d'une analyse qualitative. Une véritable articulation entre analyses qualitatives et quantitatives peut conduire les chercheurs à de nouvelles interprétations du phénomène étudié (Tashakkori \& Teddlie, 2010). Ainsi, les méthodes mixtes pourraient contribuer à faire évoluer les recherches qualitatives en intervention, en améliorant la compréhension de l'activité in situ des pratiquants ou des intervenants.

\section{Bibliographie}

Cleary, T. J., Zimmerman, B. J. \& Keating, T. (2006). Training physical education students to self- regulate during basketball free throw practice. Research Quarterly for Exercise and Sport 77(2), 251-262. https://doi.org/10.1080/ $\underline{02701367.2006 .10599358}$ 
Articuler des analyses qualitatives et quantitatives pour mieux comprendre l'activité i...

Gal-Petitfaux, N., Adé, D., Poizat, G., \& Seifert, L. (2013). L'intégration de données biomécaniques et d'expérience pour comprendre l'activité de nageurs élites et concevoir un dispositif d'évaluation. Le Travail Humain, 76(3), 257-282.

Greene, J. C. (2007). Mixed methods in social inquiry. San Franscisco: John Wiley \& Sons.

Guetterman, T. C., Fetters M. D., \& Creswell, J. W. (2015). Integrating quantitative and qualitative results in health science mixed methods research through joint displays. The Annals of Family Medicine, 13, 6, 554-561.

Hauw, D., Rochat, N., Gesbert, V., Astolfi, T., Philippe, R.A., \& Mariani, B. (2017). Putting together first-and thirdperson approaches for sport activity analysis: The case of ultra-trail runners' performance analysis. In, P. Salmon \& A.C. Macquet (Eds.), Advances in Human Factors in Sports and Outdoor Recreation. Proceedings of the AHFE 2016 International Conference on Human Factors in Sports and Outdoor Recreation, July 27-31, 2016, Walt Disney World®, Florida, USA (pp. 49-58). New York, NY: Springer.

Kermarrec, G., \& Bossard, C. (2014). Defensive Soccer Players Decision-Making: a Naturalistic Study. Journal of Cognitive Engineering and Decision Making, 8, 2, 187-199. https://doi.org/10.1177/1555343414527968

Kermarrec, G., \& Michot, T. (2007). Développement et validation d'une échelle de mesure situationnelle des stratégies d'apprentissage en contexte scolaire. Canadian Journal of Behavioural Science, 39, 3, 235-245.

Kermarrec, G., Todorovitch, J., \& Fleming, D. (2004). Investigation of the self-regulation components students employ in the physical Education setting. Journal of Teaching in Physical Education, 2, 23, 123-142.

Kerivel, T., Bossard, C., \& Kermarrec, G. (2020). Applying Inputs Process Outcomes model to team learning in sport curriculum: an exploratory qualitative study in soccer players. Journal of Human Kinetics, in press

Klein G. (2015). A Naturalistic Decision-Making perspective on studying intuitive decision - making. Journal of Applied Research in Memory and Cognition, 4, 164-168.

Le Menn, M., Kermarrec, G., Bossard, C., Travassos, B., \& Duarte, R. (2019). Intuitive Decision-Making of Expert Goalkeeping Performance: a Naturalistic Study. Journal of Human Kinetics, 69. https://doi.org/10.2478/hukin-2019-0042

Macquet, A.C. (2009). Recognition within the Decision-Making Process: A Case Study of Expert Volleyball Players. Journal of Applied Sport Psychology, 21(1), 64-80.

Bourbousson, G., Saury, J., \& Sève, C. (2012). Understanding team coordination in doubles table tennis: Joint analysis of first-and third-person data. Psychology of Sport and Exercise, 13(5), 630-639

Roure, C., Kermarrec, G., \& Pasco, D. (2019). Effects of Situational Interest Dimensions on Students' Learning Strategies in Physical Education, European Physical Education Review, 25, 2, 327-340. https://doi.org/10.1177/ $\underline{1356336 X 17732964}$

Tashakkori, A., \& Teddlie, C. (2010). Handbook of Mixed Methods in Social and Behavioral Research (2nd ed.). Thousand Oaks, CA, Sage Publication.

Theureau, J. (2010). Les entretiens d'auto-confrontation et de remise en situation par les traces matérielles et le programme de recherche « cours d'action ». Revue d'anthropologie des connaissances, 4(2), 287-322.

Vors, O., Gal-Petitfaux, N., \& Potdevin, F. (2015). A successful form of trade-off in compensatory policy classrooms. European Physical Education Review, 21(3), 340-361. https://doi.org/10.1177/1356336X15569373 
Actes de la 11ème Biennale de l'ARIS : Former des citoyens physiquement éduqués. Axe 2 - Vers une éducation physique de qualité, 10.25518/sepaps20.426

PDF automatiquement généré le 2023-04-26 11:48:49

Url de l'article : https://popups.uliege.be/sepaps20/index.php?id=426

Publié par ULiège Library en Open Access et distribué suivant les termes et les conditions de la licence CC-BY (https://creativecommons.org/licenses/by/4.0/deed.fr) 